\section{Variáveis meteorológicas e poluição do ar e sua associação com internações respiratórias em crianças: estudo de caso em São Paulo, Brasil}

\author{
Meteorological variables and air pollution and \\ their association with hospitalizations due to \\ respiratory diseases in children: a case study \\ in São Paulo, Brazil
}

\section{Variables meteorológicas y contaminación del aire y su asociación con internamientos por enfermedades respiratorias en niños: estudio de caso en São Paulo, Brasil}

\section{Resumo}

As transformações no clima urbano das cidades, a excessiva poluição atmosférica e o aumento das desigualdades sociais tornaram-se fatores determinantes do alto risco de internações por doenças respiratórias. Dessa forma, o objetivo deste trabalho foi compreender como os atributos meteorológicos (temperatura do ar, umidade relativa do ar e precipitação) e a poluição do ar (material particulado com diâmetro aerodinâmico menor de $10 \mu m-M P_{10}$ ) estão relacionados com as internações hospitalares por doenças respiratórias em crianças, em 14 distritos da cidade de São Paulo, Brasil. A combinação dos modelos lineares generalizados com uma distribuição binomial negativa e o modelo não linear distributed lag non-linear model (DLNM) foram utilizados como método estatístico para analisar a relação entre as internações, os atributos climáticos e a poluição no período de 2003 a 2013. Os resultados mostraram relações estatísticas significativas de alto risco relativo entre a temperatura média do ar $\left(17,5^{\circ} \mathrm{C}\right.$ a $21^{\circ} \mathrm{C}$, para o total analisado), umidade relativa do ar (84\% a $98 \%$ para o sexo feminino), precipitação ( $0 \mathrm{~mm}$ a $2,3 \mathrm{~mm}$ para o total e ambos os sexos e > 120mm para o sexo feminino) e $M P_{10}\left(>35 \mu \mathrm{g} / \mathrm{m}^{3}\right.$ para o total e para o sexo feminino). Com base nesses resultados, foi possivel identificar que os atributos ambientais contribuem para o elevado risco de internações.

Doenças Respiratórias; Poluição do Ar; Precipitação Atmosférica; Umidade; Temperatura Ambiente
Sara Lopes de Moraes 1

Ricardo Almendra 2

Paula Santana 2

Emerson Galvani 1

doi: 10.1590/0102-311X00101418

\author{
Correspondência \\ S. L. Moraes \\ Faculdade de Filosofia, Letras e Ciências Humanas, \\ Universidade de São Paulo. \\ Rua Carlos Koch 359, São Paulo, SP 13610-040, Brasil. \\ sara.moraes@usp.br \\ 1 Faculdade de Filosofia, Letras e Ciências Humanas, \\ Universidade de São Paulo, São Paulo, Brasil. \\ 2 Universidade de Coimbra, Coimbra, Portugal.
}




\section{Introdução}

As condições de tempo e clima sempre influenciaram de maneira direta e indireta na natureza, nas atividades e na vida cotidiana do homem. No entanto, a dinâmica climática, ambiental, bem como as condições sociais e econômicas, começaram a se modificar por conta do rápido processo de urbanização e do crescimento das cidades.

Nas grandes cidades, como é o caso da cidade de São Paulo, Brasil, que possui mais de 11 milhões de habitantes (Instituto Brasileiro de Geografia e Estatística. Censo demográfico, 2010. https://censo2010.ibge.gov.br, acessado em 05/Mar/2018), a poluição do ar, o tempo e o clima local foram as condições ambientais que mais se transformaram 1 . Tais transformações podem ser percebidas especialmente nos efeitos térmicos e na qualidade do ar, uma vez que os efeitos da ilha de calor ficaram mais intensos e a frequência de eventos extremos de temperatura aumentaram, além da excessiva emissão de poluentes na atmosfera.

Esses efeitos, acompanhados das desigualdades sociais e ambientais no espaço urbano, podem afetar a saúde e a qualidade de vida da população. Isso porque o processo de urbanização também favoreceu o surgimento e agravamento de diversas doenças 2,3 .

Um estudo realizado por Gasparrini et al. 4 em 384 lugares distribuídos em 13 países, incluindo o Brasil e a cidade de São Paulo, mostra a relação entre a temperatura ambiente, seus extremos e a mortalidade. Segundo os autores, foi identificado um efeito substancial da temperatura na mortalidade, sendo o calor e o frio responsáveis por 4,62\% no total da mortalidade para São Paulo.

A variação da temperatura do ar e de outros atributos meteorológicos, como umidade relativa do ar, precipitação e os poluentes atmosféricos, de acordo com a literatura, são as principais razões ambientais para o aumento do risco de internações e das mortes causadas por doenças respiratórias no mundo 5,6,7,8,9,10,11,12.

A umidade relativa do ar e a precipitação, assim como a temperatura e a poluição do ar, são fatores que podem afetar o funcionamento pulmonar, facilitar o surgimento de gripes, rinites, bronquites e a piora dos casos crônicos como asma, pois contribuem para o aparecimento e crescimento de vírus, bactérias, mofos, fungos e alérgenos (internos e externos) 9,11,13,14.

Atualmente, as altas taxas de mortalidade e de prevalência por doenças respiratórias, particularmente as doenças crônicas como asma e a doença pulmonar obstrutiva crônica (DPOC), se tornaram um importante problema de saúde pública. De acordo com um estudo da Carga Global de Doença (GBD) 15, a asma é a doença respiratória crônica de maior prevalência no mundo. Entretanto, mais de 3,2 milhões pessoas morreram devido à DPOC em 2015, representando um aumento de 11,6\% na mortalidade, quando comparado ao ano de 1990.

As doenças respiratórias atingem sobretudo as crianças porque são consideradas mais suscetíveis aos efeitos da mudança de tempo e da exposição à poluição atmosférica por apresentarem alta taxa metabólica e respiratória, além do fato de os mecanismos de defesa (sistema imunológico) e termorregulador estarem em desenvolvimento 5,16,17.

Apesar de as pesquisas apontarem uma relação dos efeitos dos atributos meteorológicos e da poluição do ar com o aumento do risco da mortalidade e das internações por doenças respiratórias, poucos estudos foram delineados em climas tropicais, sendo a maioria desses estudos realizados em países de clima temperado ou subtropical 7,9,11,18,19.

Portanto, o presente trabalho teve como objetivo principal investigar a relação entre as internações por doenças respiratórias em crianças com até nove anos de idade e os atributos meteorológicos (temperatura do ar, umidade relativa do ar e precipitação) e a poluição do ar em 14 distritos da cidade de São Paulo. 


\section{Materiais e métodos}

\section{Área de estudo}

A área de estudo desta pesquisa é composta por 14 distritos localizados na cidade de São Paulo, sendo eles: Belém, Bom Retiro, Brás, Casa Verde, Limão, Pari, República, Santa Cecília, Santana, Sé, Tucuruvi, Vila Guilherme, Vila Maria e Vila Medeiros. Esses distritos se localizam principalmente na Zona Norte e no centro do Município de São Paulo (Figura 1).

De acordo com o censo de 2010 do Instituto Brasileiro de Geografia e Estatística (https://cen so2010.ibge.gov.br, acessado em 05/Mar/2018), 970.663 habitantes residem nessa área, das quais 53.001 são crianças de 0 a 9 anos de idade. Estima-se que mais da metade desta população $(52,1 \%)$ utiliza o Sistema Único de Saúde (SUS) 20.

Por fim, destaca-se que a maior parte da população residente $(53,1 \%)$ na área de estudo mora em domicílios particulares permanentes com mais de 3 pessoas, e 4,38\% do rendimento nominal mensal é de apenas 1 salário mínimo e a taxa de analfabetismo nos distritos chega até $26,9 \%$ (Instituto Brasileiro de Geografia e Estatística. Censo demográfico, 2010. https://censo2010.ibge.gov.br, acessado em 05/Mar/2018).

No que diz respeito ao clima do Município de São Paulo, ele é caracterizado por faixas zonais e regiões climáticas entre o clima tropical úmido de altitude (com período seco) e subtropical (úmido ao sul da faixa pela atividade frontal), tendo como principal aspecto a alternância das estações 21 .

A temperatura média na normal climatológica de 1961 a 1990 apresenta os menores valores médios nos meses de abril a agosto, sendo a variação entre $16,7^{\circ} \mathrm{C}$ (julho) a $20,5^{\circ} \mathrm{C}$ (outubro). $\mathrm{O}$ mês mais quente é fevereiro com $23,4^{\circ} \mathrm{C}$ de temperatura média. A precipitação média mensal varia entre $238,9 \mathrm{~mm}$ em janeiro e 38,9 em agosto; a média anual da precipitação na normal é de 1.454,8mm (Instituto Nacional de Meteorologia. BDMEP - Banco de Dados Meteorológicos para Ensino e Pesquisa, 2014. http://www.inmet.gov.br/projetos/rede/pesquisa/, acessado em 20/Out/2017).

A variabilidade climática da localidade é influenciada por diversos sistemas atmosféricos como, por exemplo, a atuação do sistema anticiclone subtropical do Atlântico Sul (ASAS) que é responsável pelos períodos secos, a presença das massas de ar tropical atlântica $(\mathrm{mTa})$, massa tropical continental $(\mathrm{mTc})$, e a massa polar atlântica $(\mathrm{mPa}) 22$. Outros controles de circulação secundária como a proximidade do oceano e o relevo também são importantes na dinâmica climática local, bem como as atividades antrópicas como o processo de urbanização, uso e ocupação do solo podem interferir no aquecimento da superfície e na sensação térmica 23 .

\section{Aquisição de dados}

Para a realização desta pesquisa, foram utilizados dados secundários das variáveis meteorológicas, de poluição atmosférica e dos internamentos hospitalares durante o período de 2003 a 2013. Os dados meteorológicos diários de temperatura média do ar $\left({ }^{\circ} \mathrm{C}\right)$, precipitação $(\mathrm{mm})$ e umidade relativa do ar (\%) foram obtidos junto à estação meteorológica convencional que está localizada no distrito de Santana (2330' S; 46³7' O; altitude: 792,1 m ANMM) pertencente ao Instituto Nacional de Meteorologia (INMET), que disponibilizou os dados por meio do Banco de dados Meteorológicos para Ensino e Pesquisa (BDMEP. http://www.inmet.gov.br/projetos/rede/pesquisa/, acessado em 20/Out/2017).

As falhas $(2,61 \%)$ de umidade relativa do ar e das temperaturas médias, do ar, foram preenchidas com os dados da estação meteorológica automática do INMET (23³0' S, 46³7' O; altitude: 792,06m ANMM).

O poluente atmosférico $\mathrm{MP}_{10}$ (material particulado com diâmetro aerodinâmico menor de $10 \mu \mathrm{m}$ ) foi escolhido para representar as condições da poluição do ar, uma vez que foi considerado um poluente que colabora para o aumento da morbidade e mortalidade por doenças respiratórias 5,24. As médias diárias do $\mathrm{MP}_{10}$ foram obtidas na plataforma Qualar da Companhia Ambiental de São Paulo (CETESB. https://cetesb.sp.gov.br/ar/qualar/, acessado em 05/Mar/2018) pelas estações de monitoramento localizadas no Parque Dom Pedro II e em Santana.

Os dados epidemiológicos referentes às internações hospitalares foram obtidos de maneira pública pelos registros das Autorizações de Internação Hospitalar (AIH) do Departamento de Informática 
Figura 1

Mapa da localização da área de estudo. Município de São Paulo, Brasil.
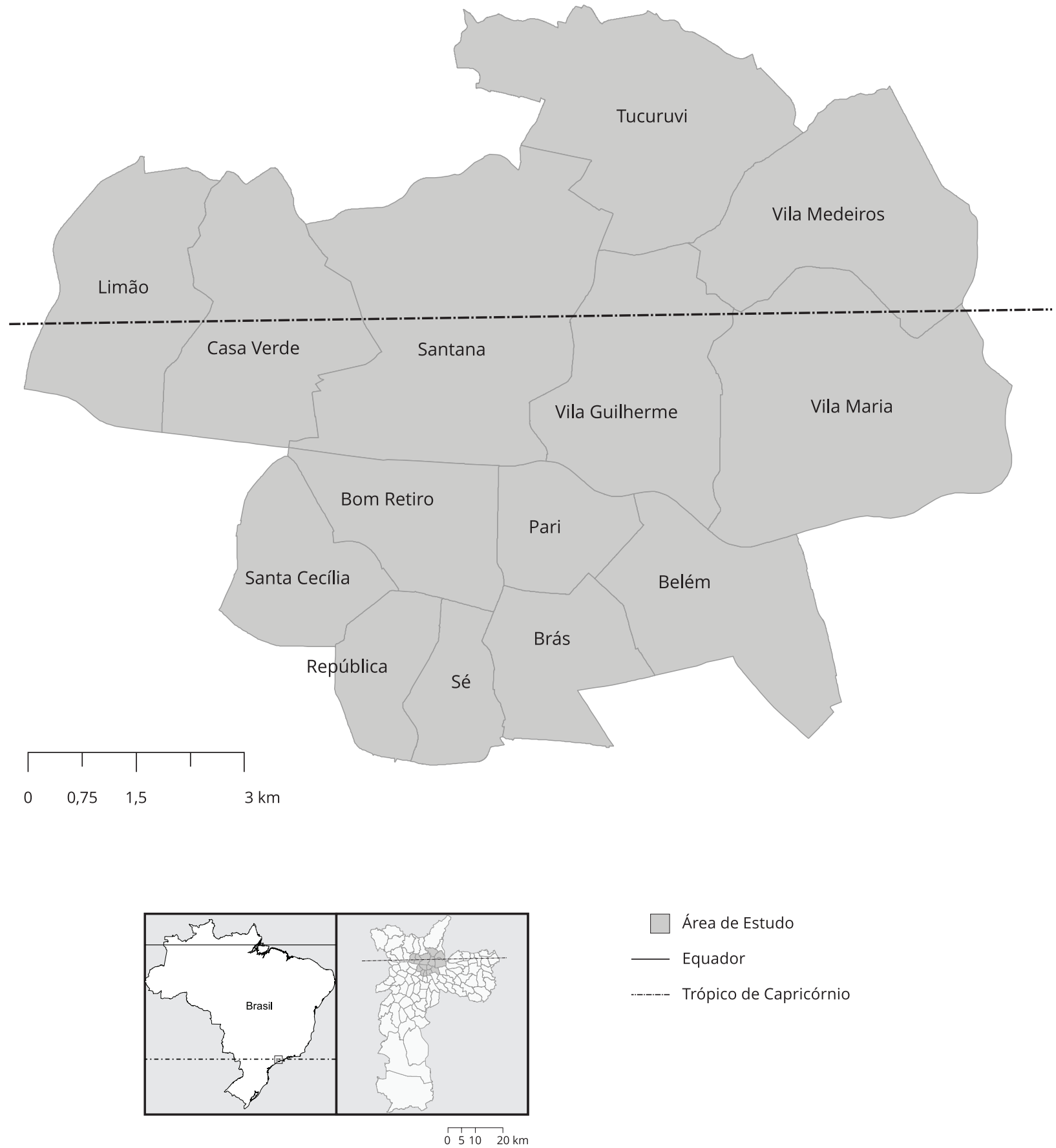

Área de Estudo

Equador

Trópico de Capricórnio

Fonte: elaboração própria, levando em consideração as bases cartográficas do Instituto Brasileiro de Geografia e Estatística (IBGE. https://downloads. ibge.gov.br/index.htm, acessado em 01/Nov/2017); Secretaria Municipal de Desenvolvimento Urbano de São Paulo (SMDU. http://www.prefeitura. sp.gov.br/cidade/secretarias/desenvolvimento_urbano/dados_estatisticos/index.php?p=160798, acessado em 01/Nov/2017) 
do Sistema Único de Saúde (DATASUS. http://www.datasus.gov.br). Foram consideradas para análise apenas as internações de crianças de 0 (zero) a 9 anos de idade que apresentaram como diagnóstico principal as doenças respiratórias (J00-J32 e J40-J47) de acordo com capítulo X da 10a revisão da Classificação Internacional de Doenças (CID-10).

A escolha deste grupo etário se baseou na literatura 5,8,10,25, já que essa faixa etária apresenta uma maior suscetibilidade às doenças do aparelho respiratório, particularmente as crianças de 0 a 5 anos. Apesar de o grupo de 6 a 9 anos apresentar características biológicas e individuais distintas das crianças menores de 5 anos, esta faixa etária foi considerada na análise, pois também há uma suscetibilidade significativa às doenças respiratórias crônicas, como a asma 25.

As doenças respiratórias (J00-J32 e J40-J47) selecionas para este estudo incluem as infecções agudas das vias aéreas superiores e inferiores, influenza, pneumonia, doenças crônicas das vias aéreas inferiores e outras doenças das vias aéreas inferiores.

\section{Análise estatística}

Para analisar a relação entre os internamentos e as variáveis de temperatura média do ar, umidade relativa do ar, precipitação e poluição do ar $\left(\mathrm{MP}_{10}\right)$, foi adotada a combinação de modelos linear generalizados (GLM) com uma distribuição binomial negativa e o modelo não linear distributed lag non-linear model (DLNM), que em conjunto permitem calcular o risco relativo (RR) acumulado de cada variável ao longo do tempo. A distribuição binomial negativa foi empregada para ajustar a sobredispersão de Poisson referente às internações.

A aplicação do DLNM permite analisar a relação das variáveis de maneira não linear ao longo do espaço da variável preditora (internações) e na dimensão de defasagem no tempo (lag), por meio da função cross-basis (cb) 26,27.

A consideração da defasagem da exposição (lag) é importante poeque as suas consequências (manifestação ou até mesmo a procura por tratamento médico) acontecem dias após a exposição a determinados eventos, como por exemplo, baixas temperaturas, alta concentração de poluentes na atmosfera, entre outros 28 .

Dessa maneira, para se calcular essa relação entre a temperatura média do ar, umidade relativa do ar, precipitação e $\mathrm{MP}_{10}$ com as internações, foi aplicado um modelo representado na equação abaixo:

$$
\begin{gathered}
\text { Yt } \sim \text { Binomial Negativa }(\mu \mathrm{t}) \\
\log (\mathrm{Yt})=\alpha+\mathrm{T}(\mathrm{df} ; \text { lag, } \mathrm{df})+\mathrm{UR}(\mathrm{df} ; \text { lag, df })+\mathrm{P}(\mathrm{df} ; \text { lag, } \mathrm{df})+ \\
\mathrm{MP}_{10}(\text { lag, } \mathrm{df})+\mathrm{NS}(\text { tempo, df })+\mathrm{DS}+\text { Feriado }
\end{gathered}
$$

Onde Y é o total das internações observadas, t representa os dias observados entre 2003 a 2013; $\mu$ t são as internações diárias esperadas; $\alpha$ é o intercepto; o lag representa a defasagem de cada variável como a temperatura média do ar $(\mathrm{T})$, umidade relativa do ar $(\mathrm{UR})$, precipitação $(\mathrm{P})$ e o poluente $\mathrm{MP}_{10}$. O tempo na equação é uma variável contínua, sendo utilizada a função splines cúbicos naturais (NS) para remover e controlar a tendência e a sazonalidade. $O$ grau de liberdade escolhido para o tempo foi o valor 7 para cada ano, e tal escolha se baseou na análise dos gráficos ACF e na literatura existente $11,28,29,30$. Além disso, os dias da semana (DS) e os feriados também foram controlados, entrando no modelo como variáveis categóricas.

A definição dos lags máximos para cada variável ambiental ( $\operatorname{T}$ lag = 28 dias; UR lag = 8; P lag = 8; e $\mathrm{MP}_{10}$ lag =1) se fundamentou em estudos anteriores e na análise de sensibilidade realizada 11,28,31. A temperatura do ar, por exemplo, é um atributo que possui efeitos distintos no organismo no período do frio e do calor, por isso foi levado em conta um tempo de defasagem mais longo para abranger tantos os efeitos das elevadas e reduzidas temperaturas $7,28 . \mathrm{O} \mathrm{MP}_{10}$, por sua vez possui efeitos mais imediatos no corpo humano, com base nisso, lags de 1 a 2 dias são mais comuns 28 .

Os demais parâmetros do modelo, como o espaço do lag e o espaço das variáveis foram definidos pela análise de sensibilidade, assim como os lags máximos. Diversos df foram testados, sendo a escolha do modelo final baseada no melhor ajuste do modelo de acordo com os valores estimados da máxima verossimilhança restrita (REML 8.541,2), e, em caso de similaridade de valores, foi aplicado o critério de parcimônia.

Portanto, o espaço da defasagem para a temperatura média do ar, umidade relativa e precipitação e $\mathrm{MP}_{10}$ foram suavizados por meio de NS com $3 \mathrm{df}$, já o espaço das variáveis para os atributos 
meteorológicas também foi modelado mediante $3 \mathrm{df}$, e, para o $\mathrm{MP}_{10}$, assumiu-se uma função linear, que também foi definida pela análise de sensibilidade.

Nos modelos não lineares, o RR é estimado para cada lag ou para o lag acumulado pela adoção de uma referência para cada variável. Para a temperatura média do ar e poluição, foi adotada a mediana como referência; para a umidade relativa do ar e precipitação, a média.

O RR foi estimado com um intervalo de 95\% de confiança (IC95\%) para todas as variáveis. Os valores dos resultados do RR podem ser interpretados da seguinte maneira: valores maiores que 1 indicam uma associação positiva ou aumento do risco relativamente ao valor de referência; valores menores que 1 são entendidos como uma relação inversa, um fator de proteção, ou seja, um menor risco; valores iguais a 1 representam ausência de associação no desfecho encontrado.

Foi efetuada uma análise estratificada por sexo, mantendo todas as opções metodológicas previamente identificadas. Os pacotes DLNM (28) e MGCV foram utilizados para a análise estatística no software R versão 3.4.2 (http://www.r-project.org).

\section{Resultados}

\section{Análise descritiva}

Durante os anos de 2003 a 2013, foram registradas 17.517 internações hospitalares por doenças respiratórias em crianças de 0 a 9 anos de idade, e mais da metade dos casos atendidos foi do sexo masculino ( $54 \%$; 9.446 casos).

Das doenças respiratórias registradas mais da metade das internações está ligada à influenza e pneumonia (56,36\%) e às doenças crônicas das vias aéreas inferiores, como asma e bronquite $(24,43 \%)$.

Na Tabela 1, é possível observar as medidas descritivas das internações totais e separadas por sexo. Nota-se que, ao longo do período estudado, o valor máximo das internações em um único dia foi de 17 casos no total. Os valores das medidas descritivas das internações referentes ao sexo masculino e ao sexo feminino são parecidos, havendo pouca variação entre os valores máximos, médias e desvio padrão.

Do mesmo modo, pode-se visualizar na tabela os valores máximos, mínimos, a média, a mediana e o desvio padrão da temperatura média do ar, umidade relativa do ar, precipitação e do poluente $\mathrm{MP}_{10}$. Ressalta-se que a precipitação tem aproximadamente $85 \%$ dos seus dados dentro do intervalo $0 \mathrm{~mm}$ a $10 \mathrm{~mm}$, em que $75 \%$ possuem valor igual a $0,0 \mathrm{~mm}$. Além disso, importa destacar que o maior valor do poluente $\mathrm{MP}_{10}$ foi de $185,4 \mu \mathrm{g} / \mathrm{m}^{3}$.

Tabela 1

Medidas descritivas das internações totais e por sexo, temperatura média do ar, umidade relativa do ar, precipitação e $\mathrm{MP}_{10}$ no período de 2003 a 2013.

\begin{tabular}{|c|c|c|c|c|c|}
\hline Variáveis & Média & Mediana & Desvio padrão & Mínimo & Máximo \\
\hline Internações & 4,4 & 4,0 & 2,6 & 0,0 & 17,0 \\
\hline Sexo feminino & 2,0 & 2,0 & 1,6 & 0,0 & 11,0 \\
\hline Sexo masculino & 2,3 & 2,0 & 1,7 & 0,0 & 10,0 \\
\hline Temperatura média do $\operatorname{ar}\left({ }^{\circ} \mathrm{C}\right)$ & 20,9 & 21,1 & 3,4 & 7,1 & 29,0 \\
\hline Umidade relativa do ar (\%) & 73,6 & 75,3 & 11,6 & 28,0 & 98,3 \\
\hline Precipitação (mm) & 4,6 & 0,0 & 11,7 & 0,0 & 140,4 \\
\hline $\mathrm{MP}_{10}\left(\mu \mathrm{g} / \mathrm{m}^{3}\right)$ & 38,2 & 33,6 & 20,2 & 5,8 & 185,4 \\
\hline
\end{tabular}




\section{Relação entre os atributos ambientais e as internações por doenças respiratórias em crianças}

Para esta pesquisa, os resultados da relação entre a temperatura média do ar, umidade relativa do ar e as internações de criança por doenças do aparelho respiratório podem ser observados na Figura 2. Os gráficos mostram o RR acumulado referente à temperatura média do ar nos lags 0-5 dias, 0-15 dias, 0-25 dias e lag máximo de 0-28 dias e à umidade relativa do ar nos lags 0-2 dias, 0-5 dias e 0-8 dias, tanto para o total das internações quanto para cada sexo. Os IC95\% estão representados pela faixa em cinza; as linhas em preto mostram o RR acumulado.

Os resultados mais significativos da relação entre a temperatura média do ar e as internações foram encontrados nas menores temperaturas (baixo RR para todos os desfechos, especialmente no lag de 5 dias) e nas temperaturas intermédias de $17,5^{\circ} \mathrm{C}$ a $21^{\circ} \mathrm{C}$, que representam um alto RR para o total analisado, sendo mais evidente a partir dos 25 dias de defasagem. Do mesmo modo, os sexos feminino e masculino apresentaram nesse intervalo de temperaturas médias um maior RR, porém

\section{Figura 2}

Relação entre a temperatura média do ar nos lags 0-5, 0-15 e 0-28, a umidade relativa do ar nos lags 0-2, 0-5 e 0-8 e as internações totais e por sexo (com intervalo de 95\% de confiança), no período de 2003 a 2013.

2a) Temperatura média do ar

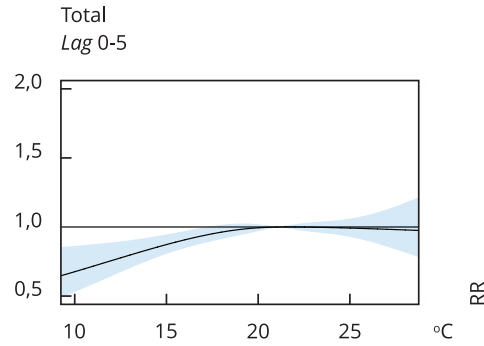

Lag 0-15

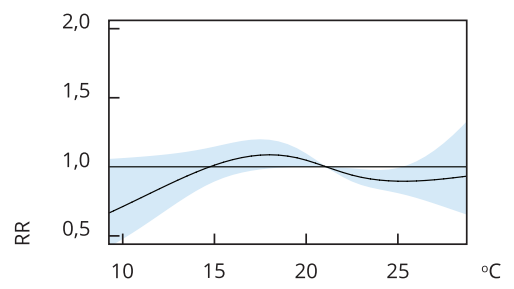

$\operatorname{Lag} 0-28$

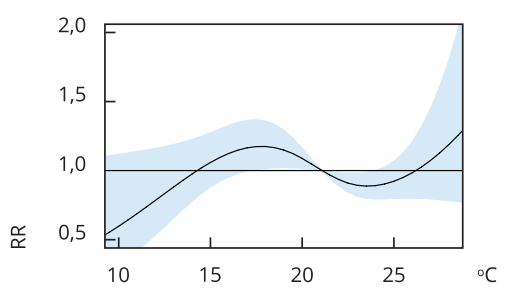

Sexo feminino

Lag 0-5

0,5

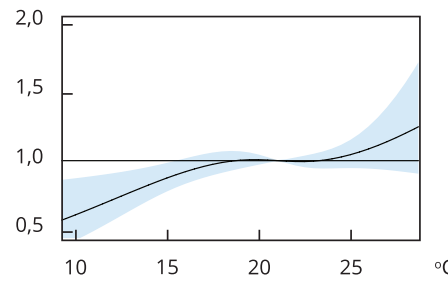

Sexo masculino

Lag 0-5

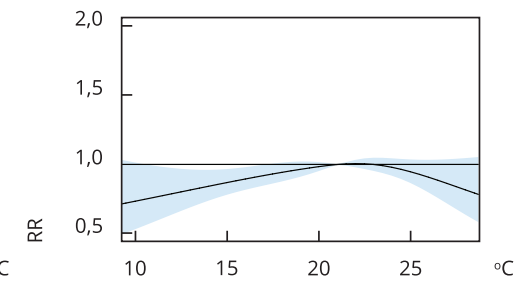

$\stackrel{\sim}{\simeq} \quad 0,5$

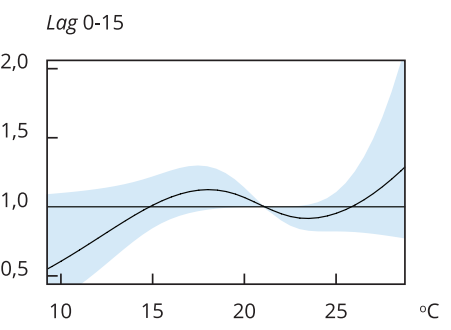

Lag 0-15

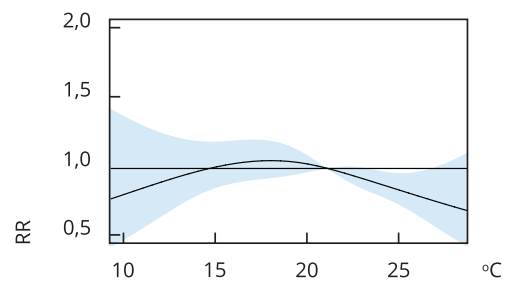

$\operatorname{Lag} 0-28$

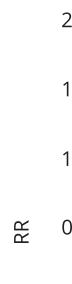

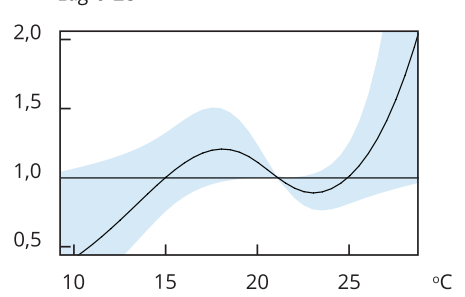

Lag 0-28

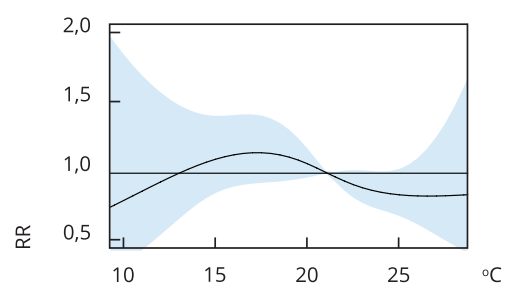

(continua) 
2b) Umidade relativa do ar
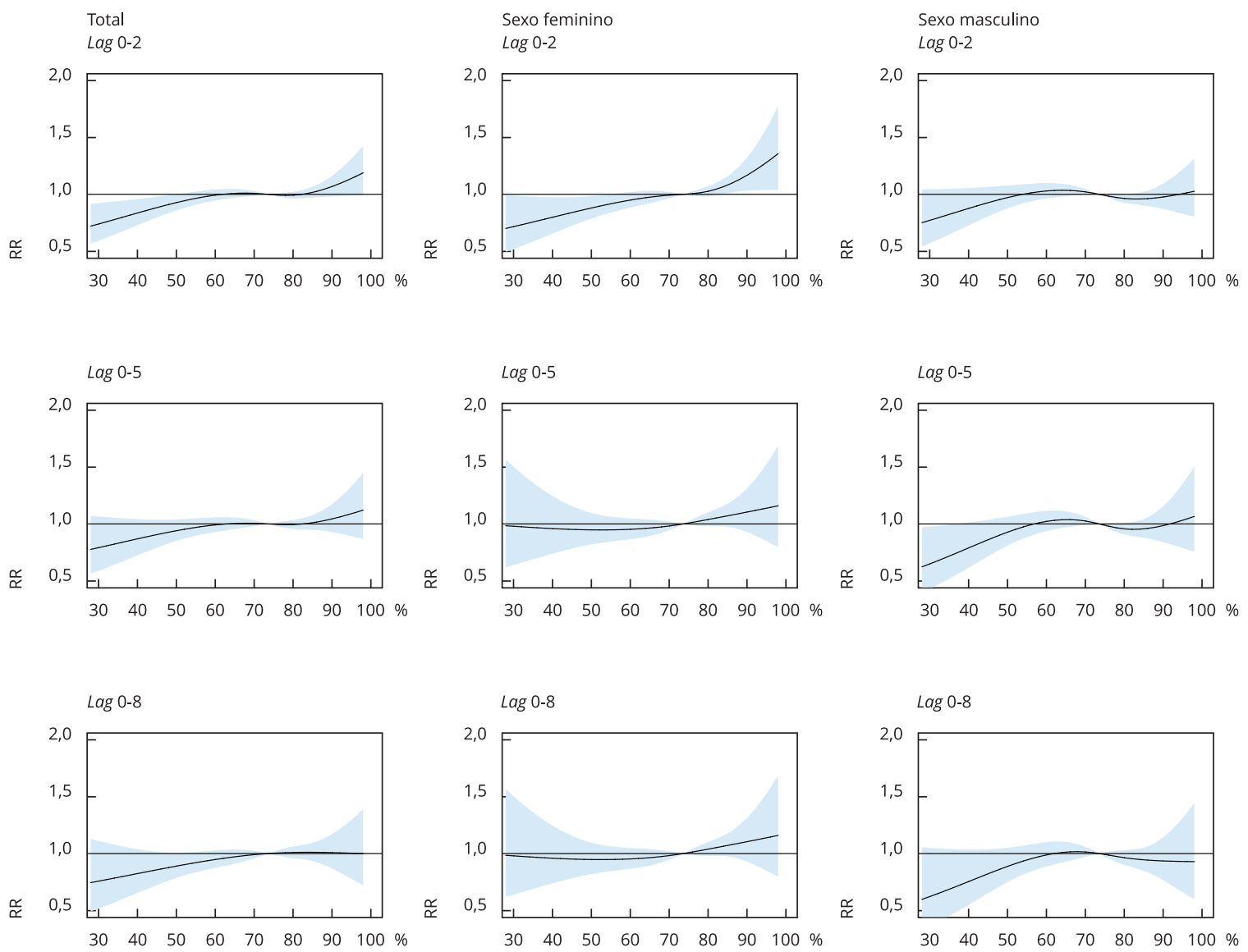

RR: risco relativo.

não foram estatisticamente significativos. $\mathrm{O}$ valor do RR significativo mais elevado foi encontrado na temperatura de $18^{\circ} \mathrm{C}(\mathrm{RR}=1,174$; IC95\%: 1,$017 ; 1,363)$.

As temperaturas mais elevadas, no entanto, representam apenas uma tendência de alto RR nas temperaturas médias do ar mais elevadas, acima de $26^{\circ} \mathrm{C}$ para as crianças do sexo feminino desde os primeiros dias de defasagem até menos expressivo do risco relativo nos últimos dias de defasagem (lag 0-25 e lag 0-28).

No que tange aos resultados relacionados à umidade relativa do ar, nota-se que os valores mais baixos de UR (28\% a 50\%) indicam menor RR nas três análises em todo o período de defasagem, exceto para o sexo feminino nos lags de 6 e 7 dias. Dessa maneira, é possível constatar que as menores porcentagens de UR não foram determinantes para o aumento das internações.

Foram encontrados resultados significativos de alto RR acumulado para as crianças do sexo feminino nas faixas mais elevadas de umidade relativa do ar (> 84\%), até os primeiros dois dias de exposição, atingindo um valor de risco máximo de 1,359 (IC95\%: 1,038; 1,778) nos 98\% de UR.

A Figura 3 apresenta os resultados da relação entre as internações, a precipitação e o poluente $\mathrm{MP}_{10}$. A precipitação foi a variável meteorológica que mostrou resultados mais significativos de aumento do RR para o total analisado e por sexo. As chuvas entre $0 \mathrm{~mm}$ a 3,0mm representam alto risco para o total das internações e para o sexo feminino durante todo o período de defasa- 


\section{Figura 3}

Relação entre a precipitação nos lags 0-2, 0-5, e 0-8, MP 10 nos lags 0-1 e as internações totais por sexo (com intervalo de 95\% de confiança), no período de 2003 a 2013.

3a) Precipitação

\section{Total}

Lag 0-2

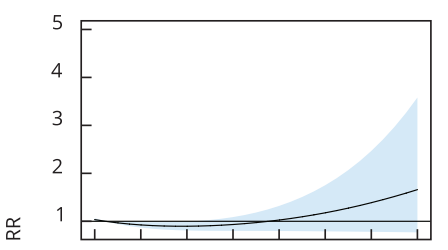

$\begin{array}{lllllllll}0 & 20 & 40 & 60 & 80 & 100 & 120 & 140 & \mathrm{~mm}\end{array}$

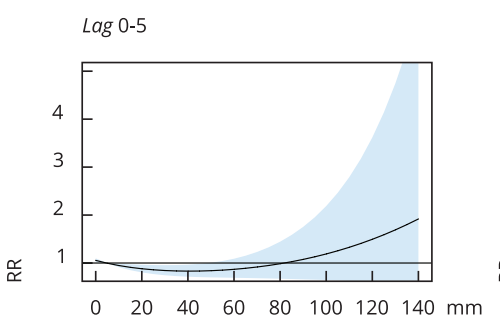

Lag 0-8

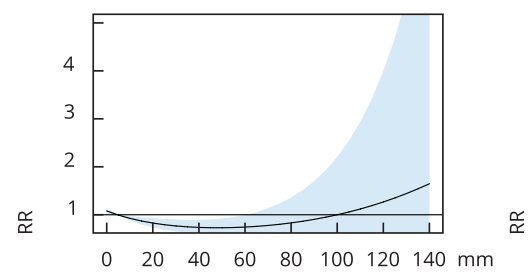

Sexo feminino

Lag 0-2

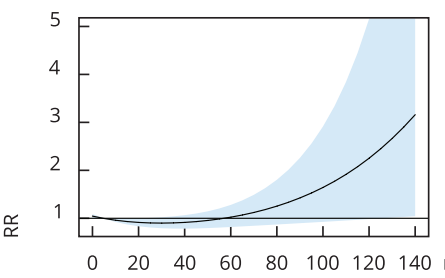

$\begin{array}{lllllllll}0 & 20 & 40 & 60 & 80 & 100 & 120 & 140 & \mathrm{~mm}\end{array}$
Sexo masculino

Lag 0-2

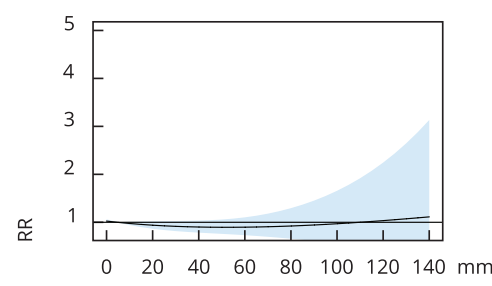

Lag 0-5

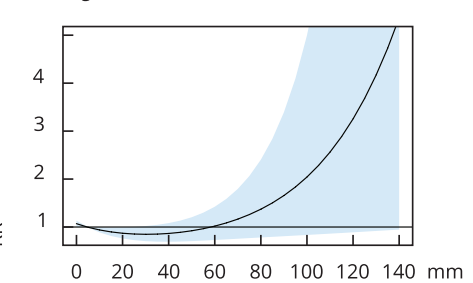

$\frac{\alpha}{\alpha}$

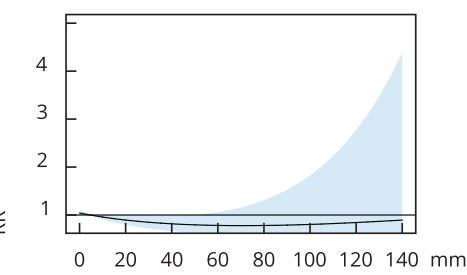

Lag 0-8

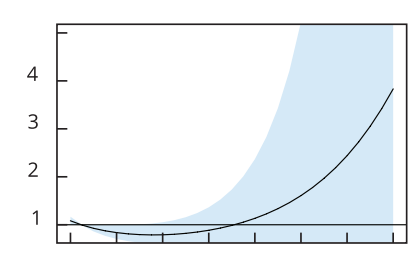

$\begin{array}{llllllll}20 & 40 & 60 & 80 & 100 & 120 & 140 & \mathrm{~mm}\end{array}$

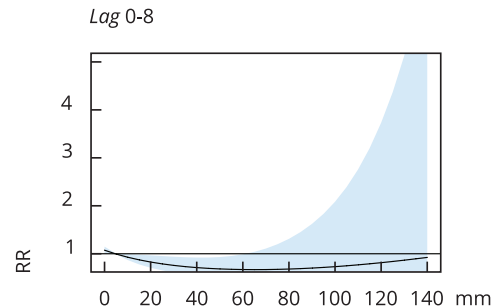

3b) Poluição do ar (MP10)
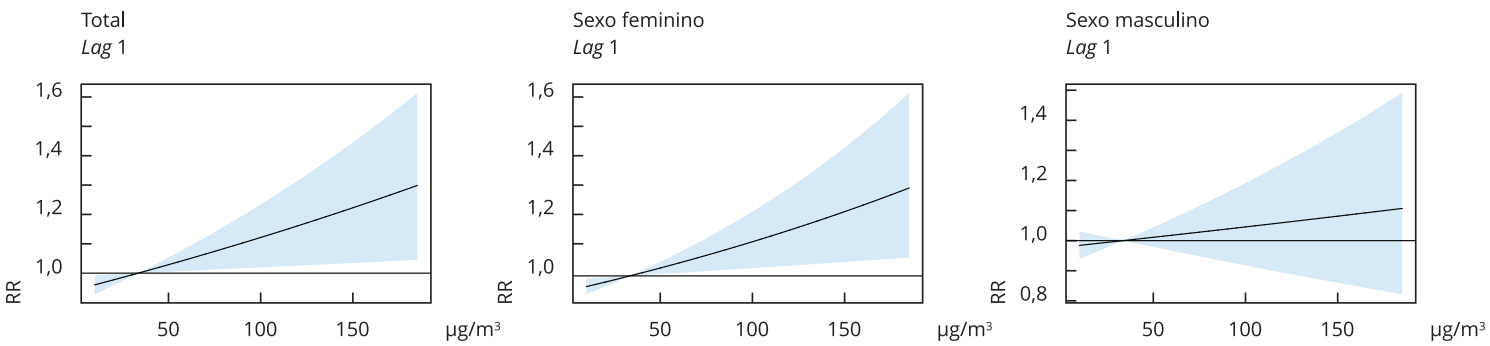
gem, enquanto para o sexo masculino o maior risco foi constatado apenas no período de exposição mais longo (lag 0-8).

Os menores riscos significativos foram encontrados a partir dos $5 \mathrm{~mm}$ de chuvas diários até $15 \mathrm{~mm}$, já o grande acúmulo de chuva $(>120 \mathrm{~mm})$ foi significativo para o aumento do RR no sexo feminino entre os lags de 2 a 4 dias, nos demais dias de defasagem também se percebeu o aumento do $\mathrm{RR}$, todavia não foram significativos estatisticamente. $\mathrm{O}$ efeito contrário aconteceu com as crianças do sexo masculino que apresentaram uma tendência ao menor risco de internações nos valores elevados de precipitação.

De acordo com os resultados de poluição $\left(\mathrm{MP}_{10}\right)$, é possível verificar, ainda, nos gráficos da Figura 3, que durante o período analisado, o alto risco relativo acumulado (significativo) relacionado ao $\mathrm{MP}_{10}$ é encontrado nas concentrações superiores a $35 \mu \mathrm{g} / \mathrm{m}^{3}$ nas crianças do sexo feminino a partir do momento da exposição e que, para o total analisado, apenas no dia seguinte à exposição. Sendo o maior RR encontrado no lag 1 no valor de 1,512 (IC95\%: 1,914; 2,067) para o sexo feminino na concentração de $185 \mu \mathrm{g} / \mathrm{m}^{3}$; já os valores abaixo de $30 \mu \mathrm{g} / \mathrm{m}^{3}$ de $\mathrm{MP}_{10}$ indicaram riscos menores para a ocorrência das internações.

A Tabela 2 representa de maneira resumida os resultados e os valores de risco relativo acumulado nos intervalos e nas defasagens mais significativas das análises entre os atributos ambientais e seu respectivo desfecho, total e por sexo.

\section{Discussão}

Os resultados deste trabalho mostraram, portanto, a relação entre cada atributo ambiental (temperatura média do ar, umidade relativa, precipitação e $\mathrm{MP}_{10}$ ) e as internações por doenças respiratórias em crianças com até 9 anos de idade, em 14 distritos localizados na cidade de São Paulo.

A temperatura do ar é considerada um dos principais atributos ambientais que contribuem para os agravos das doenças respiratórias, visto que sua variação ao longo do dia, a rápida mudança de tempo e as ocorrências de eventos extremos podem interferir no desempenho do corpo humano e deixar o ambiente mais propício às condições que auxiliam no aumento do risco de internações e da mortalidade.

As temperaturas reduzidas, por exemplo, estão relacionadas, sobretudo, com a diminuição da capacidade e da função pulmonar e com a propagação de diversos vírus, bactérias e alérgenos 32,33. As temperaturas elevadas em determinadas condições, porém, podem contribuir para que o nervo sensorial da fibra-C pulmonar reduza a passagem de ar nas vias aéreas (broncoconstrição) e cause maiores riscos de internações e mortalidade por doenças respiratórias 34 .

Além disso, o efeito do frio e do calor se manifestam em tempos diferentes no organismo, as temperaturas mais baixas tendem a ter um efeito mais prolongado e as temperaturas altas apresentam um efeito curto e imediato 7,19 .

Percebe-se que os resultados deste estudo divergem das diversas contribuições científicas $6,7,8,11,19,35$ que apontam que os efeitos da exposição ao frio, ao calor e à ocorrência de temperaturas extremas são significativos, para o aumento do risco de morte por doenças respiratórias. Cabe notar, também, essa diferença de resultados pela identificação dos padrões das curvas do risco e nos gráficos, pois as curvas da relação entre temperatura média do ar e as internações não seguem as formas de curvas do padrão U, J e V encontradas na literatura atual, especialmente nos estudos que relacionam a temperatura com a mortalidade.

Os resultados significativos do alto RR nas temperaturas intermédias podem ser considerados pela perspectiva da maior frequência de observação no outono e inverno. A variação da temperatura do ar nesta estação é influenciada pela passagem dos sistemas frontais que causam instabilidade e nebulosidade que contribuem para a queda rápida da temperatura 21,36 .

Embora não haja um consenso entre os limites de temperaturas do ar e de defasagem que de fato determinem maior risco 8 , avalia-se que a heterogeneidade de resultados possa ser atribuída a diversos fatores, como a variabilidade climática de cada lugar e a capacidade de adaptação de cada indivíduo às mudanças de tempo e clima. 


\section{Tabela 2}

Risco relativo (RR) acumulado para os intervalos mais significativos para total das internações e por sexo.

\begin{tabular}{|c|c|c|}
\hline Fatores/Intervalo */Lag & Maior RR ** & Menor RR \\
\hline \multicolumn{3}{|l|}{ Total de internações } \\
\hline \multicolumn{3}{|c|}{ Temperatura média do $\operatorname{ar}\left({ }^{\circ} \mathrm{C}\right)$} \\
\hline \multicolumn{3}{|l|}{$17,5-21$} \\
\hline $0-28$ & $1,174(1,017: 1,363)$ & $1,008(1,001 ; 1,014)$ \\
\hline \multicolumn{3}{|l|}{ Umidade relativa do ar (\%) } \\
\hline \multicolumn{3}{|l|}{$84-98$} \\
\hline $0-2$ & $1,189(0,936 ; 1,429)$ & $1,010(0,963 ; 1,053)$ \\
\hline \multicolumn{3}{|l|}{ Precipitação (mm) } \\
\hline \multicolumn{3}{|l|}{$0-2,03$} \\
\hline $0-2$ & $1,035(1,008 ; 1,063)$ & $1,017(1,004 ; 1,030)$ \\
\hline $0-5$ & $1,055(1,015 ; 1,097)$ & $1,026(1,007 ; 1,046)$ \\
\hline $0-8$ & $1,080(1,026 ; 1,136)$ & $1,038(1,013 ; 1,063)$ \\
\hline \multicolumn{3}{|l|}{$>120$} \\
\hline $0-3$ & $1,824(0,720 ; 4,618)$ & $1,464(0,731 ; 2,933)$ \\
\hline \multicolumn{3}{|l|}{$\mathrm{MP}_{10}\left(\mu \mathrm{g} / \mathrm{m}^{3}\right)$} \\
\hline \multicolumn{3}{|l|}{$>35$} \\
\hline $0-1$ & $1,299(1,045 ; 1,614)$ & $1,002(1,000 ; 1,004)$ \\
\hline \multicolumn{3}{|l|}{ Sexo feminino } \\
\hline \multicolumn{3}{|c|}{ Temperatura média do $\operatorname{ar}\left({ }^{\circ} \mathrm{C}\right)$} \\
\hline \multicolumn{3}{|l|}{$17,5-21$} \\
\hline $0-28$ & $1,209(0,971 ; 1,504)$ & $1,010(0,999 ; 1,020)$ \\
\hline \multicolumn{3}{|l|}{ Umidade relativa do ar (\%) } \\
\hline \multicolumn{3}{|l|}{$84-98$} \\
\hline $0-2$ & $1,359(1,038 ; 1,778)$ & $1,068(1,004 ; 1,136)$ \\
\hline \multicolumn{3}{|l|}{ Precipitação (mm) } \\
\hline \multicolumn{3}{|l|}{$0-2,03$} \\
\hline $0-2$ & $1,045(1,006 ; 1,087)$ & $1,021(1,003 ; 1,041)$ \\
\hline $0-5$ & $1,070(1,010 ; 1,133)$ & $1,033(1,005 ; 1,062)$ \\
\hline $0-8$ & $1,083(1,005 ; 1,167)$ & $1,039(1,002 ; 1,078)$ \\
\hline \multicolumn{3}{|l|}{$>120$} \\
\hline $0-3$ & $4,274(1,116 ; 16,373)$ & $2,777(1,017 ; 7,579)$ \\
\hline \multicolumn{3}{|l|}{$\mathrm{MP}_{10}\left(\mu \mathrm{g} / \mathrm{m}^{3}\right)$} \\
\hline \multicolumn{3}{|l|}{$>35$} \\
\hline $0-1$ & $1,512(1,914 ; 2,067)$ & $1,004(1,001 ; 1,007)$ \\
\hline \multicolumn{3}{|l|}{ Sexo masculino } \\
\hline \multicolumn{3}{|c|}{ Temperatura média do $\operatorname{ar}\left({ }^{\circ} \mathrm{C}\right)$} \\
\hline $17,5-21$ & & \\
\hline $0-28$ & $1,148(0,930 ; 1,418)$ & $1,006(0,997 ; 1,016)$ \\
\hline Umidade relativa do ar (\% & & \\
\hline $84-98$ & & \\
\hline $0-2$ & $1,027(0,802 ; 1,315)$ & $0,959(0,906 ; 1,014)$ \\
\hline Precipitação (mm) & & \\
\hline $0-2,03$ & & \\
\hline $0-2$ & $1,017(0,988 ; 1,047)$ & $1,012(0,995 ; 1,030)$ \\
\hline $0-5$ & $1,036(0,988 ; 1,086)$ & $1,020(0,995 ; 1,046)$ \\
\hline $0-8$ & $1,074(1,004 ; 1,149)$ & $1,035(1,002 ; 1,070)$ \\
\hline$>120$ & & \\
\hline $0-3$ & $0,970(0,275 ; 3,419)$ & $0,917(0,357 ; 2,353)$ \\
\hline $\mathrm{MP}_{10}\left(\mu \mathrm{g} / \mathrm{m}^{3}\right)$ & & \\
\hline$>35$ & & \\
\hline $0-1$ & $1,001(0,998 ; 1,004)$ & $1,107(0,822 ; 1,491)$ \\
\hline
\end{tabular}

* Intervalo significativo que representa alto RR;

** Maior RR acumulado encontrado no intervalo;

$\star \star \star$ Menor RR acumulado encontrado no intervalo. 
A umidade relativa, assim como a temperatura do ar, é capaz de influenciar a saúde humana, já que interfere nas sensações de conforto e desconforto térmico, que, por sua vez, estão relacionadas ao sistema termorregulador do corpo humano, que é responsável por regular/manter o equilíbrio térmico do organismo com meio ambiente 17.

A combinação de determinadas temperaturas do ar e da quantidade de umidade relativa pode colaborar para o stress térmico do corpo humano. O desconforto térmico humano pode ser sentido, sobretudo, quando a temperatura do ar está elevada e o ar apresenta alta quantidade de vapor d'água na atmosfera, ou seja, quando a umidade relativa se encontra no seu ponto de saturação 17. Essa condição leva o corpo humano a ter uma menor capacidade de retenção de água na superfície da pele, o que dificulta o resfriamento do organismo, provocando assim desconforto, estresse e fadiga no organismo 18 . Além disso, a alta umidade relativa do ar colabora para a sensibilidade a alérgenos (externos e internos), a mofos e fungos 13,14.

Em contraposição, os baixos valores de umidade relativa do ar cooperam para o ressecamento das mucosas, desidratação das células e também tornam o ambiente mais propício à transmissão de vírus e ao crescimento de mofos, fungos, bactérias e outros micro-organismos nos locais de moradia, causando alergias, rinites (alérgicas e não alérgicas), crises de asma, entre outras inflamações da membrana mucosa $13,14,37,38$.

A variação da umidade relativa do ar, por conseguinte, pode afetar o corpo humano de diversas formas. Levando isso em consideração, a Organização Mundial da Saúde (OMS) 39 classificou que os valores de umidade relativa entre 30\% e 50\% são níveis ideais para a saúde humana, os valores acima de $65 \%$ podem contribuir para o aumento de alergias, asma e doenças respiratórias do trato superior e os valores abaixo de $20 \%$ representam estados de alerta e emergência.

Islam et al. 11 encontraram resultados semelhantes ao desta pesquisa para os valores acima de 90\% na ocorrência de emergências hospitalares por doenças respiratórias do trato inferior em Londres (Inglaterra), com um lag de 0-2 dias. Já Lam et al. 9 mostraram que o aumento do risco de internações por asma em crianças de 0 a 14 anos de idade estão relacionados à alta umidade na estação mais quente.

Deve-se ter em conta, ainda, que a precipitação e a poluição do ar também são fatores importantes e podem concorrer para o aumento das internações. A precipitação, por exemplo, auxilia na "limpeza" da atmosfera, deixando-a com menor concentração de poluentes que podem colaborar com os problemas do aparelho respiratório 40. Entretanto, como foi possível observar nos resultados os eventos de elevado volume e intensidade de chuvas nos ambientes urbanos podem provocar um alto RR e diversos problemas para a população, em razão do aumento da proliferação de mofos e fungos nas moradias, particularmente nos locais mais precários que carecem de uma infraestrutura adequada 39,41.

Na cidade de Changwon (Coreia do Sul), os limites de precipitação ou de ocorrência de neve entre $5 \mathrm{~mm}$ a $15 \mathrm{~mm}$ tiveram resultados positivos para o aumento das emergências pediátricas relacionadas com as doenças respiratórias 10 . Os autores Islam et al. ${ }^{11}$ encontraram resultados significativos para o aumento de internações na cidade de Londres, quando a precipitação diária foi maior que 30mm.

No tocante à poluição do ar, verificou-se que as altas concentrações do poluente $\mathrm{MP}_{10}$ na atmosfera contribuem para o aumento do RR. Quando as concentrações de $\mathrm{MP}_{10}$ são elevadas, seus efeitos são sentidos pelo corpo humano de uma maneira mais imediata, afetando a função pulmonar, aumentando as crises de asma, bronquite e outras infecções do sistema respiratório 24 . A OMS 42 estima que a poluição do ar é responsável por 25\% das mortes por câncer de pulmão, 17\% por doenças respiratórias do trato inferior e $48 \%$ das mortes pela DPOC no mundo.

Braga et al. 5 analisaram as internações por doenças respiratórias em crianças e adolescentes em São Paulo durante janeiro de 1993 e novembro de 1997 e encontraram resultados significativos na relação entre as altas concentrações de $\mathrm{MP}_{10}\left(>35 \mu \mathrm{g} / \mathrm{m}^{3}\right)$, especialmente em crianças com até dois anos de idade. Outro estudo 43 ainda revela que o poluente $\mathrm{MP}_{10}$ aumenta em $7 \%$ a mortalidade infantil na metrópole paulistana. Constata-se, pois, que o $\mathrm{MP}_{10}$ é um poluente muito relevante quando se trata dos seus efeitos na saúde humana, principalmente nas grandes cidades que possuem como fonte principal de poluição os veículos, como é o caso de São Paulo.

Em geral, pode-se observar nos resultados que as crianças do sexo feminino possuem maior risco de internações aos atributos ambientais, do que as crianças do sexo masculino, embora seja comprovado em alguns estudos que os meninos são mais suscetíveis às doenças respiratórias por 
apresentarem diferenças na dimensão das vias aéreas, bem como na função pulmonar e imunológica nos primeiros anos de vida 44,45 .

Não obstante, não há consenso na literatura sobre as causas do maior ou menor risco entre os sexos e, consequentemente, dos intervalos mais críticos. Tais diferenças nos resultados apresentados podem associar-se a diversos fatores determinantes, como a variabilidade climática local, a capacidade de adaptação e da resposta corporal de cada indivíduo às variáveis ambientais e às mudanças climáticas. Devem-se ter conta ainda as condições socioeconômicas e habitacionais da população, pois as desigualdades e iniquidades sociais contribuem para o aumento da morbidade e mortalidade por doenças respiratórias 46,47 .

\section{Limitações}

Como esta pesquisa tem uma abordagem ecológica, as conclusões apresentadas devem ser baseadas apenas em seus objetivos. Cumpre evidenciar, ainda, que foram utilizados somente dados médios de uma única estação meteorológica, levando em conta que os valores meteorológicos e ambientais podem variar ao longo da área de estudo. Deve-se atentar ainda para a limitação dos dados epidemiológicos que abrangem exclusivamente as internações pagas pelo SUS. Apesar de as internações de crianças neonatais e menores de 1 ano de idade (correspondem a 0,46\% do total de internações) terem particularidades específicas não foram tratadas separadamente neste estudo.

\section{Considerações finais}

A realização deste estudo possibilitou a identificação dos intervalos e das defasagens cruciais da relação entre os atributos ambientais e as internações de crianças por doenças respiratórias. Nos resultados obtidos, verificou-se que o período de alto risco acontece no intervalo das temperaturas intermédias, o que difere do intervalo padrão encontrado nos dados de mortalidade pela literatura. As temperaturas extremas tanto de frio quanto de calor, portanto, não são as condições mais críticas para a ocorrência das internações na área de estudo.

Os resultados mostraram que a análise dos outros atributos ambientais, umidade relativa do ar, a precipitação e a poluição atmosférica são atributos essenciais e significativos ao entendimento do aumento das internações de crianças, especialmente as do sexo feminino que revelaram ser mais sensíveis quando expostas a determinadas condições de tempo. Por fim, estudos como este, podem contribuir para o desenvolvimento e a criação de políticas públicas adequadas que reduzam os impactos do clima na saúde humana.

\section{Colaboradores}

S. L. Moraes e R. Almendra contribuíram com a concepção e projeto, análise e interpretação dos dados, redação do artigo e revisão crítica relevante do conteúdo intelectual. P. Santana e E. Galvani participaram da concepção e projeto, revisão crítica relevante do conteúdo intelectual e aprovaram a versão final a ser publicada.

\section{Informações adicionais}

ORCID: Sara Lopes de Moraes (0000-0002-68724131); Ricardo Almendra (0000-0002-2712-9643); Paula Santana (0000-0002-7658-8475); Emerson Galvani (0000-0002-8082-5963).

\section{Agradecimentos}

S. L. Moraes e E. Galvani agradecem à Fundação de Amparo à Pesquisa do Estado de São Paulo (FAPESP; processo número: 2016/06963-3) e Coordenação de Aperfeiçoamento de Pessoal de Nível Superior (Capes) pelo financiamento da pesquisa. R. Almendra é financiado pela Fundação para a Ciência e Tecnologia (SFRH/BD/92568/2013). R. Almendra e P. Santana fazem parte do Grupo 2: Cidades e Desenvolvimento Territorial do Centro de Estudos de Geografia e Ordenamento do Território (UID/GEO/04084/2013), financiado pelo COMPETE 2020. 


\section{Referências}

1. Monteiro CA. Teoria e clima urbano. In: Monteiro CA, Mendonça F, organizadores. Clima urbano. São Paulo: Contexto; 2003. p. 9-68.

2. Ribeiro H, Vargas HC. Urbanização, globalização e saúde. Rev USP 2015; (107):13-26.

3. Ribeiro H. Patologias do ambiente urbano: desafios para a geografia da saúde. In: Lemos AIG, Silveira ML, Arroyo M, Santos M, organizadores. Questões territoriais na América Latina. São Paulo: CLACSO Livros/Departamento de Geografia, Universidade de São Paulo; 2006. p. 273-96.

4. Gasparrini A, Guo Y, Hashizume M, Lavigne E, Zanobetti A, Schwartz J, et al. Mortality risk attributable to high and low ambient temperature: a multicountry observational study. Lancet $2015 ; 386: 369-75$.

5. Braga AL, Saldiva PH, Pereira LA, Menezes JJ, Conceição GM, Lin CA, et al. Health effects of air pollution exposure on children and adolescents in São Paulo, Brazil. Pediatr Pumonol 2001; 31:106-13.

6. Sherbakov T, Malig B, Guirguis K, Gershunov A, Basu R. Ambient temperature and added heat wave effects on hospitalizations in California from 1999 to 2009. Environ Res 2018; 160:83-90

7. Muggeo VM, Hajat S. Modelling the non-linear multiple-lag effects of ambient temperature on mortality in Santiago and Palermo: a constrained segmented distributed lag approach. Occup Environ Med 2009; 66:584-91.

8. Xu Z, Huang C, Hu W, Turner LR, Su H, Tong $\mathrm{S}$. Extreme temperatures and emergency department admissions for childhood asthma in Brisbane, Australia. Occup Environ Med 2013; 70:730-5.

9. Lam HC, Li AM, Chan EY, Goggins WB. The short-term association between asthma hospitalisations, ambient temperature, other meteorological factors and air pollutants in Hong Kong: a time-series study. Thorax 2016; 71:1097-109.

10. Lee HJ, Jin MH, Lee JH. The association of weather on pediatric emergency department visits in Changwon, Korea (2005-2014). Sci Total Environ 2016; 551-552:699-705.

11. Islam MS, Chaussalet TJ, Koizumi N. Towards a threshold climate for emergency lower respiratory hospital admissions. Environ Res 2017; 153:41-7.

12. O'Lenick CR, Chang HH, Kramer MR, Winquist A, Mulholland JA, Friberg MD, et al. Ozone and childhood respiratory disease in three US cities: evaluation of effect measure modification by neighborhood socioeconomic status using a Bayesian hierarchical approach. Environ Health 2017; 16:36.

13. Tischer CG, Heinrich J. Exposure assessment of residential mould, fungi and microbial components in relation to children's health: achievements and challenges. Int J Hyg Environ Health 2013; 216:109-14.
14. Tham R, Katelaris CH, Vicendese D, Dharmage SC, Lowe AJ, Bowatte G, et al. The role of outdoor fungi on asthma hospital admissions in children and adolescents: a 5-year time stratified case-crossover analysis. Environ Res 2017; 154:42-9.

15. GBD 2015 Chronic Respiratory Disease Collaborators. Global, regional, and national deaths, prevalence, disability-adjusted life years, and years lived with disability for chronic obstructive pulmonary disease and asthma, 1990-2015: a systematic analysis for the Global Burden of Disease Study 2015. Lancet Respir Med 2017; 5:691-706.

16. Bunyavanich S, Landrigan CP, McMichael AJ, Epstein PR. The impact of climate change on child health. Ambul Pediatr 2003; 3:44-52.

17. Hanna EG, Tait PW. Limitations to thermoregulation and acclimatization challenge human adaptation to global warming. Int J Environ Res Public Health 2015; 12:8034-74.

18. Tang J, Xiao CC, Li YR, Zhang J, Zhai HY, Geng XY, et al. Effects of diurnal temperature range on mortality in Hefei city, China. Int J Biometeorol 2018; 62:851-60.

19. O'Lenick CR, Winquist A, Chang HH, Kramer MR, Mulholland JA, Grundstein A, et al. Evaluation of individual and area-level factors as modifiers of the association between warmseason temperature and pediatric asthma morbidity in Atlanta, GA. Environ Res 2017; 156:132-44.

20. Coordenação de Epidemiologia e Informação, Prefeitura de São Paulo. Estimativa da população exclusivamente usuária SUS no município de São Paulo. Boletim Eletrônico CEInfo 2010 1. http://www.prefeitura.sp.gov.br/cidade/se cretarias/upload/saude/arquivos/boletimele tronico/n01popsus.pdf.

21. Monteiro CA. A frente polar atlântica e as chuvas de inverno na fachada sul-oriental do Brasil: contribuição metodológica à análise rítmica dos tipos de tempo no Brasil. São Paulo: Universidade de São Paulo; 1969.

22. Nimer E. Climatologia do Brasil. 2a Ed. Rio de Janeiro: Instituto Brasileiro de Geografia e Estatística; 1989.

23. Tarifa JR, Armani G. Os climas urbanos. In: Tarifa JR, Azevedo TR, organizadores. Os climas na cidade de São Paulo: teoria e prática. São Paulo: GeoUSP; 2001. p. 47-70.

24. Pope CA, Dockery DW. Health effects of fine particulate air pollution: lines that connect. J Air Waste Manag Assoc 2006; 56:709-42.

25. Dick S, Friend A, Dynes K, Al Kandari F, Doust E, Cowie $\mathrm{H}$, et al. A systematic review of associations between environmental exposures and development of asthma in children aged up to 9 years. BMJ Open 2014; 4:e006554.

26. Gasparrini A. Distributed lag linear and nonlinear models in R: the package dlnm. J Stat Softw 2011; 43:1-20. 
27. Gasparrini A. Modeling exposure-lag-response associations with distributed lag nonlinear models. Stat Med 2014; 33:881-99.

28. Gasparrini A, Armstrong B, Kenward MG. Distributed lag non-linear models. Stat Med 2010; 29:2224-34.

29. Li YR, Xiao CC, Li J, Tang J, Geng XY, Cui LJ, et al. Association between air pollution and upper respiratory tract infection in hospital outpatients aged 0 e 14 years in Hefei, China: a time series study. Public Health 2018; 156:92100.

30. Almendra R, Loureiro A, Silva G, Vasconcelos J, Santana P. Short-term impacts of air temperature on hospitalizations for mental disorders in Lisbon. Sci Total Environ 2019; 647:127-33.

31. Dang TN, Seposo XT, Duc NH, Thang TB, An DD, Hang LT, et al. Characterizing the relationship between temperature and mortality in tropical and subtropical cities: a distributed lag non-linear model analysis in Hue, Viet Nam, 2009-2013. Glob Health Action 2016; 9:28738.

32. Xu Z, Etzel RA, Su H, Huang C, Guo Y, Tong S. Impact of ambient temperature on children's health: a systematic review. Environ Res 2012; 117:120-31.

33. Koskela H, Tukiainen H, Kononoff A, Pekkarinen $\mathrm{H}$. Effect of whole-body exposure to cold and wind on lung function in asthmatic patients. Chest 1994; 105:1728-31.

34. Hayes D Jr, Collins PB, Khosravi M, Lin RL, Lee LY. Bronchoconstriction triggered by breathing hot humid air in patients with asthma role of cholinergic reflex. Am J Respir Crit Care Med 2012; 185:1190-6.

35. Son JY, Gouveia N, Bravo MA, Freitas CU, Bell ML. The impact of temperature on mortality in a subtropical city: effects of cold, heat, and heat waves in São Paulo, Brazil. Int J Biometeorol 2016; 60:113-21.

36. Galvani E, Azevedo TR. A frente polar atlântica e as características de tempo associadas: estudo de caso. In: Galvani E, Lima NGB, organizadores. Climatologia aplicada: resgate aos estudos de caso. Curitiba: CRV; 2012. p. 7-18.

37. Tham R, Vicendese D, Dharmage SC, Hyndman RJ, Newbigin E, Lewis E, et al. Associations between outdoor fungal spores and childhood and adolescent asthma hospitalizations. J Allergy Clin Immunol 2017; 139:1140-7.
38. Tang JW. The effect of environmental parameters on the survival of airborne infectious agents. J R Soc Interface 2009; 6 Suppl 6:S73746.

39. World Health Organization. WHO guidelines for indoor air quality: dampness and mould. Geneva: World Health Organization; 2009.

40. Garcia-Chevesich PA, Alvarado S, Neary DG, Valdes R, Valdes J, Aguirre JJ, et al. Respiratory disease and particulate air pollution in Santiago Chile: contribution of erosion particles from fine sediments. Environ Pollut 2014; 187:202-5.

41. Norbäck D, Zock JP, Plana E, Heinrich J, Tischer C, Jacobsen Bertelsen R, et al. Building dampness and mold in European homes in relation to climate, building characteristics and socio-economic status: The European Community Respiratory Health Survey ECRHS II. Indoor Air 2017; 27:921-32.

42. World Health Organization. Chronic respiratory diseases. https://www.who.int/respira tory/en (acessado em 27/Dez/2017).

43. Conceição GM, Miraglia SG, Kishi HS, Saldiva $\mathrm{PH}$, Singer JM. Air pollution and child mortality: a time-series study in São Paulo, Brazil. Environ Health Perspect 2001; 109 Suppl 3:347-50.

44. Falagas ME, Mourtzoukou EG, Vardakas KZ. Sex differences in the incidence and severity of respiratory tract infections. Respir Med 2007; 101:1845-63.

45. Becklake MR, Kauffmann F. Gender differences in airway behaviour over the human life span. Thorax 1999; 54:1119-38.

46. Marí-Dell'Olmo M, Gotsens M, Palència L, Burström B, Corman D, Costa G, et al. Socioeconomic inequalities in cause-specific mortality in 15 European cities. J Epidemiol Community Health 2015; 69:432-41.

47. Grigsby M, Siddharthan T, Chowdhury MA, Siddiquee A, Rubinstein A, Sobrino E, et al. Socioeconomic status and COPD among lowand middle-income countries. Int J Chron Obstruct Pulmon Dis 2016; 11:2497-507. 


\section{Abstract}

Urban climate changes, excessive air pollution, and increasing social inequalities have become determinant factors in the high risk of hospitalizations due to respiratory diseases. The current study thus aimed to understand how meteorological factors (air temperature, relative humidity, and precipitation) and air pollution (particulate matter with aerodynamic diameter less than $\left.10 \mu \mathrm{m}-P M_{10}\right)$ are related to hospitalizations due to respiratory diseases in children in 14 districts in the city of São Paulo, Brazil. The combination of generalized linear models with a negative binomial distribution and distributed lag non-linear model (DLNM) were used as the statistical method to analyze the relationship between hospitalizations, climatic factors, and pollution from 2003 to 2013. The results show statistically significant association with high relative risk between mean air temperature $\left(17.5^{\circ} \mathrm{C}\right.$ to $21^{\circ} \mathrm{C}$, for the total analyzed), relative humidity ( $84 \%$ to $98 \%$ for females), precipitation $(0 \mathrm{~mm}$ to $2.3 \mathrm{~mm}$ for the total and both sexes and $>120 \mathrm{~mm}$ for females), and $P M_{10}$ (> $35 \mu \mathrm{g} / \mathrm{m}^{3}$ for the total and for females). These results showed that environmental factors contribute to the high risk of hospitalizations.

Respiratory Tract Diseases; Air Pollution; Atmospheric Preciptation; Humidity; Temperature

\section{Resumen}

Las transformaciones en el clima urbano de las ciudades, la excesiva contaminación atmosférica $y$ el aumento de las desigualdades sociales se convirtieron en factores determinantes para el alto riesgo de internamientos por enfermedades respiratorias. Por ello, el objetivo de este trabajo ha sido comprender cómo las condiciones meteorológicas (temperatura del aire, humedad relativa del aire y precipitaciones) y la contaminación del aire (material particulado con un diámetro aerodinámico menor de $10 \mu m-M P_{10}$ ) están relacionados con internamientos hospitalarios por enfermedades respiratorias en niños, en 14 distritos de la ciudad de Sao Paulo. La combinación de los modelos lineales generalizados con una distribución binomial negativa y el modelo no lineal distributed lag non-linear model (DLNM) se utilizaron como método estadístico para analizar la relación entre los internamientos, atributos climáticos y la contaminación durante el periodo de 2003 a 2013. Los resultados mostraron relaciones estadísticas significativas de alto riesgo relativo entre la temperatura media del aire $\left(17,5^{\circ} \mathrm{C}\right.$ a $21^{\circ} \mathrm{C}$, para el total analizado), humedad relativa del aire (84\% a 98\% para el sexo femenino), precipitaciones $(0 \mathrm{~mm}$ a 2,3mm para el total y ambos sexos y $>120 \mathrm{~mm}$ para el sexo femenino) y $\mathrm{MP}_{10}(>35 \mu \mathrm{g} /$ $\mathrm{m}^{3}$ para el total y sexo femenino). A partir de estos resultados, fue posible identificar que los atributos ambientales contribuyen al aumento del riesgo de internamientos.

Enfermedades Respiratorias; Contaminación del Aire; Precipitación Atmosférica; Umedad; Temperatura Ambiental
Recebido em 29/Mai/2018

Versão final respresentada em 19/Jan/2019 Aprovado em 28/Jan/2019 